\title{
TIGHTNESS FOR MAXIMA OF GENERALIZED BRANCHING RANDOM WALKS
}

\author{
MING FANG, ${ }^{*}$ University of Minnesota
}

\begin{abstract}
We study generalized branching random walks on the real line $\mathbb{R}$ that allow time dependence and local dependence between siblings. Specifically, starting from one particle at time 0 , the system evolves such that each particle lives for one unit amount of time, gives birth independently to a random number of offspring according to some branching law, and dies. The offspring from a single particle are assumed to move to new locations on $\mathbb{R}$ according to some joint displacement distribution; the branching laws and displacement distributions depend on time. At time $n, F_{n}(\cdot)$ is used to denote the distribution function of the position of the rightmost particle in generation $n$. Under appropriate tail assumptions on the branching laws and offspring displacement distributions, we prove that $F_{n}\left(\cdot-\operatorname{Med}\left(F_{n}\right)\right)$ is tight in $n$, where $\operatorname{Med}\left(F_{n}\right)$ is the median of $F_{n}$. The main part of the argument is to demonstrate the exponential decay of the right tail $1-F_{n}\left(\cdot-\operatorname{Med}\left(F_{n}\right)\right)$.
\end{abstract}

Keywords: Branching random walk; recursion; tightness

2010 Mathematics Subject Classification: Primary 60G50

Secondary $60 \mathrm{~J} 80$

\section{Introduction}

We study the maximal displacement of generalized branching random walks (GBRWs), which are governed by a family of branching rules $\left\{p_{n, k}\right\}_{n \geq 0, k \geq 1}$ and displacement laws $\left\{G_{n, k}\right\}_{n \geq 0, k \geq 1}$. For this class, we assume that the $p_{n, k}$ are nonnegative real numbers such that $\sum_{k=1}^{\infty} p_{n, k}=1$ and $\sum_{k=1}^{\infty} k p_{n, k}<\infty$ for each $n \geq 0$; the $G_{n, k}$ are distribution functions on $\mathbb{R}^{k}$ for each $n$ and $k$. The GBRW is defined recursively as follows. At time 0 , a particle $o=\overline{1}$ is located at 0 . Suppose that, at time $n, v=\overline{1 \alpha_{1} \cdots \alpha_{n}}\left(\alpha_{i} \in \mathbb{N}\right)$ is a particle at location $S_{v}$. At time $n+1, v$ dies and gives birth to $K_{v} \geq 1$ (random) offspring. (We do not consider the case $K_{v}=0$ because if the system dies out then the distribution of its maximum is trivial.) We denote the offspring of $v$ at generation $n+1$ by $\left\{\overline{v 1}, \ldots, \overline{v K_{v}}\right\}$ (for labels $u$ and $v, u v$ denotes the concatenation of $u$ and $v$ ) and their locations by $\left\{S_{v}+X_{v, 1}, \ldots, S_{v}+X_{v, K_{v}}\right\}$. Let $\mathbb{D}$ be the collection of all the particles at any time and let $\mathbb{D}_{n}$ be those alive at time $n$. We consider the case where the random vectors $\left\{\left(K_{v}, X_{v, 1}, \ldots, X_{v, K_{v}}\right)\right\}_{v \in \mathbb{D}}$ indexed by particles are independent and have distributions

$$
\mathrm{P}\left(K_{v}=k \mid v \in \mathbb{D}_{n}, \mathcal{F}_{n}\right)=p_{n, k}
$$

and

$$
\begin{aligned}
& \mathrm{P}\left(X_{v, 1} \leq x_{1}, \ldots, X_{v, K_{v}} \leq x_{K_{v}} \mid v \in \mathbb{D}_{n}, K_{v}=k, \mathcal{F}_{n}\right) \\
& \quad=G_{n, k}\left(x_{1}, \ldots, x_{k}\right) \text { for } n=0,1, \ldots \text { and } k=1,2, \ldots,
\end{aligned}
$$

where $\mathcal{F}_{n}=\sigma\left\{S_{u} \mid u \in \mathbb{D}_{k}, k=0,1, \ldots, n\right\}$ is the $\sigma$-field generated by the GBRW by time $n$.

Received 27 September 2011; revision received 17 January 2012.

* Current address: School of Mathematical Sciences, Xiamen University, Xiamen, Fujian, 361005, China.

Email address: fang0086@umn.edu 
We are interested in the maximal displacement of particles at time $n$, i.e. $\mathcal{M}_{n}=\max _{v \in \mathbb{D}_{n}} S_{v}$. Let $F_{n}(x)=\mathrm{P}\left(\mathcal{M}_{n} \leq x\right)$ be the distribution function of $\mathcal{M}_{n}$, and set $\bar{F}_{n}(x)=1-F_{n}(x)$. Under some assumptions, we want to prove the tightness of the sequence of recentered distributions $F_{n}\left(\cdot-\operatorname{Med}\left(F_{n}\right)\right)$, where $\operatorname{Med}\left(F_{n}\right)$ is the median of $F_{n}$. See Section 2 and Section 5 for two different sets of assumptions under which tightness can be proved.

From the previous description, our GBRW allows time dependence (through the $n$ parameter) and some local dependence (through the joint distribution $G_{n, k}$ ). We will review some of the existing literature on tightness and make some comparisons with this paper. Dekking and Host [5] gave a short proof for tightness of $F_{n}\left(\cdot-\operatorname{Med}\left(F_{n}\right)\right)$ when the offspring displacements are all bounded above by a uniform constant. Using moment arguments, Addario-Berry and Reed [1] proved that $\mathcal{M}_{n}-\mathrm{E} \mathcal{M}_{n}$ is exponentially tight when the offspring displacements are independent and identically distributed (i.i.d.) and satisfy appropriate large deviation assumptions. Bramson and Zeitouni [4] proved the tightness of maxima of modified branching random walks derived from a Gaussian free field by modifying the arguments in [1], [2], and [5].

Using a different approach, Bramson and Zeitouni [3] provided an analytic method to prove tightness of the maximal displacement when the offspring displacement distributions depend on time and satisfy certain tail conditions; they assumed that the offspring displacements are i.i.d. and used a recursion to derive their results. When the joint distribution is locally dependent, this recursion (see (3) below) loses some of its nice properties; we will therefore not be able to apply this approach directly. Rather, it needs to be modified to take advantage of some recursion bounds; see (5) below.

In order to find a recursion, we need to look at GBRWs starting from particles at some intermediate time. For any integer $m$ and $v=\overline{1 \alpha_{1} \cdots \alpha_{m}} \in \mathbb{D}_{m}$, the process

$$
\left\{S_{u}-S_{v} \mid u=\overline{1 \alpha_{1} \cdots \alpha_{m} \beta_{1} \cdots \beta_{j}} \in \mathbb{D}_{m+j}, \beta_{j} \in \mathbb{N}, j=1,2, \ldots\right\}
$$

is a GBRW governed by the branching rules $\left\{p_{m+j, k}\right\}_{j \geq 0, k \geq 1}$ and the displacement laws $\left\{G_{m+j, k}\right\}_{j \geq 0, k \geq 1}$. For $n>m$, the maximal displacement from $S_{v}$ at time $n-m$ is denoted by $\mathcal{M}_{n}^{v}$. The $\left\{\mathcal{M}_{n}^{v}\right\}_{v \in \mathbb{D}_{m}}$ are i.i.d. random variables whose distribution is denoted by $F_{n}^{m}(\cdot)$. Again, set $\bar{F}_{n}^{m}(\cdot)=1-F_{n}^{m}(\cdot)$. Note that $F_{n}(\cdot)=F_{n}^{0}(\cdot), \bar{F}_{n}(\cdot)=\bar{F}_{n}^{0}(\cdot)$, and $\bar{F}_{n}^{n}(\cdot)=\mathbf{1}_{\{x<0\}}(\cdot)$.

We obtain a recursion for $F_{n}^{m}(\cdot)$ by looking at the first generation of GBRWs starting from particles at time $m$. For $n>m$,

$$
F_{n}^{m}(x)=\sum_{k=1}^{\infty} p_{m, k} \int_{\mathbb{R}^{k}} \prod_{i=1}^{k} F_{n}^{m+1}\left(x-y_{i}\right) \mathrm{d}^{k} G_{m, k}\left(y_{1}, \ldots, y_{k}\right) .
$$

Following [3], we consider a recursion for the tail distribution $\bar{F}_{n}^{m}(\cdot)$. For $n>m$, the above equation is equivalent to

$$
\bar{F}_{n}^{m}(x)=1-\sum_{k=1}^{\infty} p_{m, k} \int_{\mathbb{R}^{k}} \prod_{i=1}^{k}\left(1-\bar{F}_{n}^{m+1}\left(x-y_{i}\right)\right) \mathrm{d}^{k} G_{m, k}\left(y_{1}, \ldots, y_{k}\right) .
$$

Without loss of generality, for any $n, k>0$, we assume that $G_{n, k}$ has the same marginal distributions, i.e.

$$
g_{n, k}(x)=\int_{\mathbb{R}^{k-1}} \mathrm{~d}^{k-1} G_{n, k}\left(y_{1}, \ldots, y_{i-1}, x, y_{i+1}, \ldots, y_{k}\right) \quad \text { for any } 1 \leq i \leq k .
$$


Otherwise, we can replace $G_{n, k}$ by $\tilde{G}_{n, k}$ defined by

$$
\tilde{G}_{n, k}\left(x_{1}, \ldots, x_{k}\right)=\frac{1}{k !} \sum_{\pi \in \mathcal{P}_{k}} G_{n, k}\left(x_{\pi(1)}, \ldots, x_{\pi(k)}\right),
$$

where $\mathcal{P}_{k}$ denotes all the permutations on $\{1, \ldots, k\}$. Then $\tilde{G}_{n, k}$ has the same marginal distributions and we can easily check that recursion (3) is the same for $G_{n, k}$ and $\tilde{G}_{n, k}$.

To apply an approach similar to [3], we introduce two functions:

$$
Q_{1, k}(u)=1-(1-u)^{k} \quad \text { and } \quad Q_{2, k}(u)=k u \quad \text { for } 0 \leq u \leq 1 .
$$

We will work with the following recursion inequalities derived from (3), instead of (3) itself.

Lemma 1. Assume that $\bar{F}_{n}^{m}(x)$ satisfies recursion (3); then the following recursion bounds hold for $n>m$ :

$$
\sum_{k=1}^{\infty} p_{m, k} g_{m, k} * Q_{1, k}\left(\bar{F}_{n}^{m+1}\right)(x) \leq \bar{F}_{n}^{m}(x) \leq \sum_{k=1}^{\infty} p_{m, k} g_{m, k} * Q_{2, k}\left(\bar{F}_{n}^{m+1}\right)(x) .
$$

Here ' $*$ ' is the convolution defined by $g * f(x)=\int_{-\infty}^{\infty} f(x-y) \mathrm{d} g(y)$ for any two functions $f(x)$ and $g(x)$ whenever the integral makes sense.

Proof. We begin by proving the upper bound in (5). Rewrite (3) as

$$
\bar{F}_{n}^{m}(x)=\sum_{k=1}^{\infty} p_{m, k} \int_{\mathbb{R}^{k}}\left(1-\prod_{i=1}^{k}\left(1-\bar{F}_{n}^{m+1}\left(x-y_{i}\right)\right)\right) \mathrm{d}^{k} G_{m, k}\left(y_{1}, \ldots, y_{k}\right) .
$$

Using the inequality

$$
1-\prod_{i=1}^{k}\left(1-x_{i}\right) \leq \sum_{i=1}^{k} x_{i} \quad \text { for } 0 \leq x_{i} \leq 1
$$

and the fact that $G_{m, k}(\cdot, \ldots, \cdot)$ has the same marginal distributions $g_{m, k}(\cdot)$, we find that the above quantity is at most

$$
\sum_{k=1}^{\infty} p_{m, k} \int_{\mathbb{R}^{k}} \sum_{i=1}^{k} \bar{F}_{n}^{m+1}\left(x-y_{i}\right) \mathrm{d}^{k} G_{m, k}\left(y_{1}, \ldots, y_{k}\right)=\sum_{k=1}^{\infty} p_{m, k} \int_{\mathbb{R}} k \bar{F}_{n}^{m+1}(x-y) \mathrm{d} g_{m, k}(y) .
$$

Together with the definition of $Q_{2, k}$, cf. (4), we obtain the upper bound in (5).

We next prove the lower bound in (5). Applying a generalized Hölder's inequality to (3), we obtain

$$
\bar{F}_{n}^{m}(x) \geq 1-\sum_{k=1}^{\infty} p_{m, k} \prod_{i=1}^{k}\left(\int_{\mathbb{R}^{k}}\left(1-\bar{F}_{n}^{m+1}\left(x-y_{i}\right)\right)^{k} \mathrm{~d}^{k} G_{m, k}\left(y_{1}, \ldots, y_{k}\right)\right)^{1 / k} .
$$

Again, since $G_{m, k}(\cdot, \ldots, \cdot)$ possesses the same marginal distributions $g_{m, k}(\cdot)$, the right-hand 
side above equals

$$
\begin{aligned}
1-\sum_{k=1}^{\infty} p_{m, k} \prod_{i=1}^{k}\left(\int_{\mathbb{R}}\left(1-\bar{F}_{n}^{m+1}(x-y)\right)^{k} \mathrm{~d} g_{m, k}(y)\right)^{1 / k} \\
=1-\sum_{k=1}^{\infty} p_{m, k}\left(\int_{\mathbb{R}}\left(1-\bar{F}_{n}^{m+1}(x-y)\right)^{k} \mathrm{~d} g_{m, k}(y)\right) \\
=\sum_{k=1}^{\infty} p_{m, k}\left(\int_{\mathbb{R}}\left(1-\left(1-\bar{F}_{n}^{m+1}(x-y)\right)^{k}\right) \mathrm{d} g_{m, k}(y)\right) .
\end{aligned}
$$

Together with the definition of $Q_{1, k}$, see (4), we obtain the lower bound in (5).

\section{Assumptions and statement of the result for bounded branching}

In this section we discuss the tightness property in the case where the offspring number is uniformly bounded. To state our result, we need some assumptions on both the branching and displacement laws. We first introduce assumptions concerning the branching mechanism.

(B1) $\left\{p_{n, k}\right\}_{n \geq 0}$ possess a uniformly bounded support, i.e. there exists an integer $k_{0}>1$ such that $p_{n, k}=0$ for all $n$ and $k \notin\left\{1, \ldots, k_{0}\right\}$.

(B2) The mean offspring number is uniformly greater than 1 by some fixed constant. That is, there exists a real number $m_{0}>1 \operatorname{such}$ that $\inf _{n}\left\{\sum_{k=1}^{k_{0}} k p_{n, k}\right\}>m_{0}$.

We introduce the following assumptions on the displacement laws $G_{n, k}$ for those $n$ and $k$ such that $p_{n, k} \neq 0$.

(MT1) For some fixed $\varepsilon_{0}<\frac{1}{4} \log m_{0} \wedge 1$, there exists an $x_{0}$ such that $\bar{g}_{n, k}\left(x_{0}\right) \geq 1-\varepsilon_{0}$ for all $n$ and $k$, where $\bar{g}_{n, k}(x)=1-g_{n, k}(x)$. By shifting, we may and will assume that $x_{0}=0$, that is, $\bar{g}_{n, k}(0) \geq 1-\varepsilon_{0}$.

(MT2) There exist $a>0$ and $M_{0}>0$ such that $\bar{g}_{n, k}(x+M) \leq \mathrm{e}^{-a M} \bar{g}_{n, k}(x)$ for all $n, k$ and $M>M_{0}, x \geq 0$.

(GT) For any $\eta_{1}>0$, there exists a $B>0$ such that $G_{n, k}(B, \ldots, B) \geq 1-\eta_{1}$ and $G_{n, k}\left([-B, \infty)^{k}\right) \geq 1-\eta_{1}$ for all $n$ and $k$. (With an abuse of notation, $G_{n, k}$ is also used here as a function on measurable sets defined by $G_{n, k}(A):=\int_{A} \mathrm{~d}^{k} G_{n, k}\left(x_{1}, \ldots, x_{k}\right)$ for $A \subset \mathbb{R}^{k}$. See (2) for the definition of $G_{n, k}$ as a distribution function on $\mathbb{R}^{k}$.)

Assumptions (MT1) and (MT2) concern the marginal distributions. Assumption (MT1) prevents too much mass drifting to $-\infty$, while (MT2) guarantees that the right tails of the marginals decay at least exponentially. Assumption (GT) concerns the tightness of the joint distribution of the increments and prevents any step from being too negative or too positive to dominate the walk. Note that without the assumption $x_{0}=0$, (MT1) is implied by (GT). Now we are ready to state our main theorem.

Theorem 1. Under assumptions (B1), (B2), (MT2), and (GT), the family of the recentered maxima distributions $\left\{F_{n}\left(\cdot-\operatorname{Med}\left(F_{n}\right)\right)\right\}_{n \geq 0}$ is tight.

Theorem 1 is proved in Section 3, with the proofs of some propositions deferred to Section 4. With an analysis of a Lyapunov function, we control the right tails of distributions $F_{n}\left(\cdot-\operatorname{Med}\left(F_{n}\right)\right)$. Then we use assumption $(\mathrm{GT})$ together with the right tail property to control 
the behavior of the left tails of the distributions. Using a similar approach, we also prove a variation of Theorem 1 under slightly different assumptions in Section 5.

\section{A Lyapunov function, main induction, and proof of Theorem 1}

This section follows [3], with some minor revisions, in introducing a Lyapunov function. Namely, for choices of $\varepsilon_{1}, b, M>0$ (to be determined later), we define the Lyapunov function $L(\cdot)$ as

$$
L(u)=\sup _{\{x: u(x) \in(0,1 / 2]\}} l(u ; x),
$$

where

$$
l(u ; x)=\log \left(\frac{1}{u(x)}\right)+\log _{b}\left(1+\varepsilon_{1}-\frac{u(x-M)}{u(x)}\right)_{+} .
$$

Here $(x)_{+}=x \vee 0$, and we use the convention that $\log 0=-\infty$.

As in [3], the heart of the proof is contained in the following proposition.

Proposition 1. Under assumptions (B1), (B2), (MT1), and (MT2), there are choices of $\varepsilon_{1}, b$, and $M$ such that $\sup _{\{m, n: m \leq n\}} L\left(\bar{F}_{n}^{m}\right)<C$ for some finite number $C>0$.

The proof of Proposition 1 will occupy the bulk of the paper, and is detailed in Section 4. Before proving it, we discuss its consequences. As in [3, Corollary 2.8], Proposition 1 implies the following result.

Corollary 1. Suppose that assumptions (B1), (B2), (MT1), and (MT2) hold. Then there exists $\delta_{1}>0$ such that, for all $n$ and $m \leq n$, and all $x$,

$$
\bar{F}_{n}^{m}(x) \leq \delta_{1} \quad \Longrightarrow \quad \bar{F}_{n}^{m}(x-M) \geq\left(1+\frac{\varepsilon_{1}}{2}\right) \bar{F}_{n}^{m}(x) .
$$

Proof. Let $C_{0}=\sup _{m, n: m \leq n} L\left(\bar{F}_{n}^{m}\right)$. Assumptions (B1), (B2), (MT1), and (MT2) hold, so $C_{0}<\infty$ by Proposition 1. By definition (6), for all $n$ and $m$ such that $m \leq n$ and $x$ such that $0<\bar{F}_{n}^{m}(x) \leq \frac{1}{2}$,

$$
\log \left(\frac{1}{\bar{F}_{n}^{m}(x)}\right)+\log _{b}\left(1+\varepsilon_{1}-\frac{\bar{F}_{n}^{m}(x-M)}{\bar{F}_{n}^{m}(x)}\right)_{+} \leq C_{0} .
$$

This is equivalent to

$$
\frac{\bar{F}_{n}^{m}(x-M)}{\bar{F}_{n}^{m}(x)} \geq 1+\varepsilon_{1}-b^{C_{0}+\log \bar{F}_{n}^{m}(x)}
$$

The lemma follows immediately.

This corollary gives the desired control over the behavior of the right tail of $\bar{F}_{n}^{m}(\cdot)$. We next control the left tail. First, we obtain the following pointwise bounds for the integral in (3).

Lemma 2. Assumption $(G T)$ implies that, for any $\eta_{1}>0$, there exists a $B$ such that

$$
Q_{m}\left(\bar{F}_{n}^{m+1}\right)(x+B)-\eta_{1} \leq \bar{F}_{n}^{m}(x) \leq Q_{m}\left(\bar{F}_{n}^{m+1}\right)(x-B)+\eta_{1},
$$

where $Q_{m}(u)=\sum_{k=1}^{\infty} p_{m, k}\left(1-(1-u)^{k}\right)$.

Proof. The upper bound is obtained by considering only the integral over $(-\infty, B]^{k}$ in (3):

$$
\bar{F}_{n}^{m}(x) \leq 1-\sum_{k=1}^{\infty} p_{m, k} \int_{(-\infty, B]^{k}} \prod_{i=1}^{k}\left(1-\bar{F}_{n}^{m+1}\left(x-y_{i}\right)\right) \mathrm{d}^{k} G_{m, k}\left(y_{1}, \ldots, y_{k}\right) .
$$


By the monotonicity of $\bar{F}_{n}^{m+1}(\cdot)$, the right-hand side is less than

$$
1-\sum_{k=1}^{\infty} p_{m, k}\left(1-\bar{F}_{n}^{m+1}(x-B)\right)^{k} G_{m, k}(B, \ldots, B) .
$$

For any $\eta_{1}>0$, choose $B$ as in assumption (GT). Then $G_{m, k}(B, \ldots, B) \geq 1-\eta_{1}$, and the above quantity is less than or equal to

$$
\begin{aligned}
& 1-\sum_{k=1}^{\infty} p_{m, k}\left(1-\bar{F}_{n}^{m+1}(x-B)\right)^{k}\left(1-\eta_{1}\right) \\
& \quad=Q_{m}\left(\bar{F}_{n}^{m+1}\right)(x-B)+\eta_{1} \sum_{k=1}^{\infty} p_{m, k}\left(1-\bar{F}_{n}^{m+1}(x-B)\right)^{k} \\
& \quad \leq Q_{m}\left(\bar{F}_{n}^{m+1}\right)(x-B)+\eta_{1},
\end{aligned}
$$

proving the upper bound in (9). To obtain the lower bound, first rewrite (3) as

$$
\bar{F}_{n}^{m}(x)=\sum_{k=1}^{\infty} p_{m, k} \int_{\mathbb{R}^{k}}\left(1-\prod_{i=1}^{k}\left(1-\bar{F}_{n}^{m+1}\left(x-y_{i}\right)\right)\right) \mathrm{d}^{k} G_{m, k}\left(y_{1}, \ldots, y_{k}\right) .
$$

By restricting the above integral to $[-B, \infty)^{k}$, we obtain a lower bound on $\bar{F}_{n}^{m}$ :

$$
\bar{F}_{n}^{m}(x) \geq \sum_{k=1}^{\infty} p_{m, k} \int_{[-B, \infty)^{k}}\left(1-\prod_{i=1}^{k}\left(1-\bar{F}_{n}^{m+1}\left(x-y_{i}\right)\right)\right) \mathrm{d}^{k} G_{m, k}\left(y_{1}, \ldots, y_{k}\right) .
$$

Since $\bar{F}_{n}^{m+1}(x)$ is decreasing in $x$ and $G_{m, k}\left([-B, \infty)^{k}\right) \geq 1-\eta_{1}$ as in assumption (GT), we have

$$
\begin{aligned}
\bar{F}_{n}^{m}(x) & \geq \sum_{k=1}^{\infty} p_{m, k}\left(1-\left(1-\bar{F}_{n}^{m+1}(x+B)\right)^{k}\right) G_{m, k}\left([-B, \infty)^{k}\right) \\
& \geq \sum_{k=1}^{\infty} p_{m, k}\left(1-\left(1-\bar{F}_{n}^{m+1}(x+B)\right)^{k}\right)\left(1-\eta_{1}\right) \\
& =Q_{m}\left(\bar{F}_{n}^{m+1}\right)(x+B)-\eta_{1} \sum_{k=1}^{\infty} p_{m, k}\left(1-\left(1-\bar{F}_{n}^{m+1}(x+B)\right)^{k}\right) \\
& \geq Q_{m}\left(\bar{F}_{n}^{m+1}\right)(x+B)-\eta_{1},
\end{aligned}
$$

proving the lower bound in (9) and completing the proof of Lemma 2.

Lemma 2 almost verifies [3, Assumption 2.4], except that $Q_{m}$ depends on $m$. However, with assumption (B1), $Q_{m}$ satisfies [3, Properties (T1) and (T2) in Definition 2.3] uniformly in $m$. Namely, the family of strictly increasing functions $Q_{m}:[0,1] \rightarrow[0,1]$, with $Q_{m}(0)=0$ and $Q_{m}(1)=1$, satisfies the following properties.

(T1') $k_{0} x>Q_{m}(x)>x$ for all $x \in(0,1)$. For any $\delta>0$, we can choose $c_{\delta}=1+\left(m_{0}-\right.$ 1) $\delta / k_{0}>1$ such that $Q_{m}(x)>c_{\delta} x$ for all $x \in(0, \delta]$ and all $m$. 
$\left(\mathrm{T} 2^{\prime}\right)$ For each $\delta \in(0,1)$, there exists a nonnegative function $g_{\delta}(\varepsilon) \rightarrow 0$ as $\varepsilon \rightarrow 0$ (for example, choose $\left.g_{\delta}(\varepsilon)=(1-\delta)^{-k_{0}+1} \varepsilon\right)$ such that, for any $m$, if $x \geq \delta$ and $Q_{m}\left(\left(1+g_{\delta}(\varepsilon)\right) x\right) \leq$ $1-\delta$, then $Q_{m}\left(\left(1+g_{\delta}(\varepsilon)\right) x\right) \geq(1+\varepsilon) Q_{m}(x)$.

To check the above two properties, we use the strict convexity of $1-(1-x)^{k}$ and its monotonicity in $k$. Details are omitted here. From $\left(\mathrm{T}^{\prime}\right)$ and $\left(\mathrm{T}_{2}^{\prime}\right)$, we can deduce the following lemma in exactly the same way as in [3, Lemma 2.10].

Lemma 3. Suppose that (8) holds for all $m$ and $n$ such that $m \leq n$ under some choices of $\delta_{1}, M, \varepsilon_{1}>0$. Also, suppose that (B1), (B2), and (9) hold. For fixed $\eta_{0} \in(0,1)$, there exists a constant $\gamma=\gamma\left(\eta_{0}\right)<1$ and a continuous function $f(t)=f_{\eta_{0}}(t):[0,1] \rightarrow[0,1]$, with $f(t) \rightarrow 0$ as $t \rightarrow 0$ such that, for any $\varepsilon \in\left(0,\left(1-\eta_{0}\right) / \eta_{0}\right), \eta \in\left[\delta_{1}, \eta_{0}\right]$ (note that we can, without loss of generality, choose $\delta_{1}$ as small as we like; in particular, we may suppose that $\left.\delta_{1}<\eta_{0}\right)$, and large enough $N_{1}=N_{1}(\varepsilon)$, the following statement holds. If $M^{\prime}>M$ and, for any $m$ and $n$ such that $m<n, \bar{F}_{n}^{m}(x) \geq \delta_{1}$,

$$
\bar{F}_{n}^{m}\left(x-M^{\prime}\right) \leq(1+\varepsilon) \bar{F}_{n}^{m}(x), \quad \text { and } \quad \bar{F}_{n}^{m}\left(x-M^{\prime}\right) \leq \eta,
$$

then

$$
\bar{F}_{n}^{m+1}\left(x+N_{1}-M^{\prime}\right) \leq(1+f(\varepsilon)) \bar{F}_{n}^{m+1}\left(x-N_{1}\right)
$$

and

$$
\bar{F}_{n}^{m+1}\left(x+N_{1}-M^{\prime}\right) \leq \gamma \eta
$$

Proof. Assuming that (10) holds, we first prove (11). For any $\eta_{1}>0$ (to be determined later), by Lemma 2 , there exists a $B=B\left(\eta_{1}\right)\left(N_{1}=B\left(\eta_{1}\right)\right.$ for some appropriate $\left.\eta_{1}\right)$ such that

$$
\bar{F}_{n}^{m}\left(x-M^{\prime}\right) \geq Q_{m}\left(\bar{F}_{n}^{m+1}\right)\left(x-M^{\prime}+B\right)-\eta_{1}
$$

and

$$
\bar{F}_{n}^{m}(x) \leq Q_{m}\left(\bar{F}_{n}^{m+1}\right)(x-B)+\eta_{1} .
$$

Since $\bar{F}_{n}^{m}(x) \geq \delta_{1},(14)$ implies that

$$
Q_{m}\left(\bar{F}_{n}^{m+1}\right)(x-B) \geq \bar{F}_{n}^{m}(x)-\eta_{1} \geq\left(1-\frac{\eta_{1}}{\delta_{1}}\right) \bar{F}_{n}^{m}(x) ;
$$

(10) and (13) imply that

$$
\begin{aligned}
(1+\varepsilon) \bar{F}_{n}^{m}(x) & \geq \bar{F}_{n}^{m}\left(x-M^{\prime}\right) \\
& \geq Q_{m}\left(\bar{F}_{n}^{m+1}\right)\left(x-M^{\prime}+B\right)-\eta_{1} \\
& \geq Q_{m}\left(\bar{F}_{n}^{m+1}\right)\left(x-M^{\prime}+B\right)-\frac{\eta_{1}}{\delta_{1}} \bar{F}_{n}^{m}(x) .
\end{aligned}
$$

It follows from the above two inequalities that, for $\eta_{1}<\delta_{1}$,

$$
\frac{1+\varepsilon+\eta_{1} / \delta_{1}}{1-\eta_{1} / \delta_{1}} Q_{m}\left(\bar{F}_{n}^{m+1}\right)(x-B) \geq Q_{m}\left(\bar{F}_{n}^{m+1}\right)\left(x-M^{\prime}+B\right) .
$$

Let $\eta_{1}$ (which will be chosen even smaller later) be small enough so that $\left(1+\varepsilon+\eta_{1} / \delta_{1}\right) /(1-$ $\left.\eta_{1} / \delta_{1}\right) \leq 1+2 \varepsilon$. Then

$$
(1+2 \varepsilon) Q_{m}\left(\bar{F}_{n}^{m+1}\right)(x-B) \geq Q_{m}\left(\bar{F}_{n}^{m+1}\right)\left(x-M^{\prime}+B\right) .
$$


Choose $N_{1}=B$ corresponding to $\eta_{1}$. We will use property (T2') to prove (11). Since $\bar{F}_{n}^{m}(x) \geq$ $\delta_{1},(14)$ implies that

$$
Q_{m}\left(\bar{F}_{n}^{m+1}\right)(x-B) \geq \delta_{1}-\eta_{1} \geq \frac{\delta_{1}}{2},
$$

if we let $\eta_{1}<\delta_{1} / 2$. From (10) and (13),

$$
Q_{m}\left(\bar{F}_{n}^{m+1}\right)\left(x-M^{\prime}+B\right) \leq \eta+\eta_{1} \leq 1-\frac{1-\eta}{2},
$$

if we let $\eta_{1}<(1-\eta) / 2$. Define $\delta^{\prime}=\delta_{1} / 2 k_{0} \wedge(1-\eta) / 2>0$. Then

$$
\bar{F}_{n}^{m+1}(x-B) \geq \delta^{\prime} \quad \text { and } \quad Q_{m}\left(\bar{F}_{n}^{m+1}\right)\left(x-M^{\prime}+B\right) \leq 1-\delta^{\prime} .
$$

Choose $f(\varepsilon):=g_{\delta^{\prime}}(2 \varepsilon) \rightarrow 0$ as $\varepsilon \rightarrow 0$, where $g_{\delta^{\prime}}(\cdot)$ is defined in $\left(\mathrm{T} 2^{\prime}\right)$.

Now suppose that (11) is violated, i.e.

$$
\left(1+g_{\delta^{\prime}}(2 \varepsilon)\right) \bar{F}_{n}^{m+1}(x-B)<\bar{F}_{n}^{m+1}\left(x+B-M^{\prime}\right) .
$$

By the monotonicity of $Q_{m}$,

$$
Q_{m}\left(\left(1+g_{\delta^{\prime}}(2 \varepsilon)\right) \bar{F}_{n}^{m+1}(x-B)\right)<Q_{m}\left(\bar{F}_{n}^{m+1}\left(x+B-M^{\prime}\right)\right) \leq 1-\delta^{\prime} .
$$

Then, by the monotonicity of $Q_{m}$ and (T2'),

$Q_{m}\left(\bar{F}_{n}^{m+1}\left(x+B-M^{\prime}\right)\right)>Q_{m}\left(\left(1+g_{\delta^{\prime}}(2 \varepsilon)\right) \bar{F}_{n}^{m+1}(x-B)\right) \geq(1+2 \varepsilon) Q_{m}\left(\bar{F}_{n}^{m+1}\right)(x-B)$.

This contradicts (15), and (11) is proved.

To prove (12), we first obtain, by (10) and (13),

$$
Q_{m}\left(\bar{F}_{n}^{m+1}\right)\left(x-M^{\prime}+B\right) \leq \bar{F}_{n}^{m}\left(x-M^{\prime}\right)+\eta_{1} \leq \eta+\eta_{1},
$$

which indicates that

$$
\bar{F}_{n}^{m+1}\left(x-M^{\prime}+B\right) \leq \frac{Q_{m}^{-1}\left(\eta+\eta_{1}\right)}{\eta} \eta .
$$

Owing to assumption (B2), we can choose $\eta_{1}$ small enough so that

$$
\gamma:=\sup _{m} \sup _{\eta \in\left[\delta_{1}, \eta_{0}\right]} \frac{Q_{m}^{-1}\left(\eta+\eta_{1}\right)}{\eta}<1 .
$$

Therefore, (12) holds and the lemma is proved.

By iterating, the above lemma gives a connection between the left and right tail behaviors. That is, by applying Corollary 1 and Lemma 3 several times as in [3, Proof of Proposition 2.9], the same contrapositive argument proves that, for fixed $\eta_{0} \in(0,1)$, there exists an $\hat{\varepsilon}_{0}=$ $\hat{\varepsilon}_{0}\left(\eta_{0}\right)>0$, an $n_{0}$, and an $\hat{M}$ such that, if $n>n_{0}$ and $\bar{F}_{n}^{0}(x-\hat{M}) \leq \eta_{0}$, then $\bar{F}_{n}^{0}(x-\hat{M}) \geq$ $\left(1+\hat{\varepsilon}_{0}\right) \bar{F}_{n}^{0}(x)$. This will yield the following tightness proposition by recalling that $F_{n}(\cdot)=$ $F_{n}^{0}(\cdot)$.

Proposition 2. Suppose that (8) holds for all $m \leq n$ under some choices of $\delta_{1}, M, \varepsilon_{1}>0$. Also, suppose that assumption (B1) and (9) hold. Then the family of recentered maxima distributions $\left\{F_{n}\left(\cdot-\operatorname{Med}\left(F_{n}\right)\right)\right\}_{n \geq 0}$ is tight.

This completes the proof of Theorem 1, under the assumption that Proposition 1 holds. Thus, it remains to prove Proposition 1, which we do in the next section. 


\section{Analysis of the Lyapunov function and proof of Proposition 1}

In this section we focus on proving Proposition 1, which is an analog of [3, Theorem 2.7]. The same idea works here, i.e. the exponential decay of $g_{n, k}$ will not bring much mass from far away during the recursion. However, the exact approach does not quite apply here. Bramson and Zeitouni [3] dealt with the nonlinearity and convolution in a recursion equality separately. In our case, recursion (3) does not possess such a nice form. Fortunately, we have the recursion inequalities (5). These bounds require one to analyze the nonlinearity and convolution together. Throughout this section, all the sums about $k$ are from 1 to $k_{0}$ since we suppose that assumption (B1) holds. We begin with some properties of the two functions in (4). The function $Q_{2, k}(u)=$ $k u$ is simple, and the following straightforward facts about $Q_{1, k}(u)$ will be used later on.

Lemma 4. There exists a $c_{1}=c_{1}\left(k_{0}\right) \geq 1$ such that, for all $1 \leq k \leq k_{0}$ and $0 \leq u \leq 1$,

$$
Q_{1, k}(u) \geq u
$$

and

$$
k u-c_{1} u^{2} \leq Q_{1, k}(u) \leq k u=Q_{2, k}(u) .
$$

Inequality (16) follows from the fact that $Q_{1, k}(u)$ is increasing in $k$ for $0 \leq u \leq 1$. The inequalities in (17) can be easily checked by noting that $Q_{1, k}(u)=k u+$ higher order terms.

Next, we state the choices of $\varepsilon_{1}, b$, and $M$ in the Lyapunov function under which Proposition 1 holds. Throughout, we fix $k_{0}, m_{0}, \varepsilon_{0}, M_{0}$, and $a$ as in assumptions (B1), (B2), (MT1), and (MT2). Next, we choose small $\varepsilon_{1}<\frac{1}{100}, b>1$ close to 1 , large $M>100$, and an auxiliary small variable $\kappa<\frac{1}{100}$ (used later to control the flatness change) such that the following restrictions hold:

$$
\begin{gathered}
M>4 M_{0} \quad \text { and } \quad \mathrm{e}^{-a M / 2} \leq\left(4 k_{0}\right)^{4} \mathrm{e}^{-a M / 4} \leq \frac{1}{100}, \\
\frac{8\left(2 k_{0}\right)^{5 / 2} \varepsilon_{1}^{1 / 2 \log b-3 / 2}}{\left(1-\varepsilon_{0}\right) \kappa^{3 / 2}}<\frac{1}{2 c_{1}}, \\
c_{1} \frac{1+\varepsilon_{1}}{1-\varepsilon_{0}} \varepsilon_{1}^{1 / \log b} \leq \sum_{k=1}^{k_{0}} k p_{n, k}-m_{0} \quad \text { for all } n, \\
\frac{\log m_{0}}{2} \geq 2\left(\varepsilon_{1}+\varepsilon_{0}\right)+\frac{6 \kappa}{\log b}, \\
\frac{a M}{16 \log b} \geq 2\left(\varepsilon_{1}+\varepsilon_{0}+\log \left(4 k_{0}\right)\right)-\frac{\log \kappa}{\log b}, \\
\frac{a}{16 \log b} \geq \frac{2 \log \left(4 k_{0}\right)}{M} .
\end{gathered}
$$

The above conditions are compatible. In fact, thinking of $\kappa$ as $\beta \log b$, we can choose $\varepsilon_{1}$ and $\beta$ small enough so that (21) holds due to the choice of $\varepsilon_{0}$ in assumption (MT1), then we can choose $b$ close enough to 1 so that (19), (20), and (23) hold due to the choice of $m_{0}$ in assumption (B2), and, finally, we can choose $M$ large enough so that (18) and (22) hold.

With the above choices of $\varepsilon_{1}, b, M$, and $\kappa$, we can now prove Proposition 1.

Proof of Proposition 1. Choose $C=\log 2$. The conclusion $\sup _{\{m, n: m \leq n\}} L\left(\bar{F}_{n}^{m}\right) \leq C$ will follow from the claim that

$$
L\left(\bar{F}_{n}^{m}\right)>C \quad \Longrightarrow \quad L\left(\bar{F}_{n}^{m+1}\right)>C \quad \text { for any } m<n .
$$


Suppose that the conclusion is violated; then $L\left(\bar{F}_{n}^{m}\right)>C$ for some $m \leq n$. Iterating the claim $n-m$ times, we obtain $L\left(\bar{F}_{n}^{n}\right)>C$. However, $L\left(\bar{F}_{n}^{n}\right)=-\infty$ because $\bar{F}_{n}^{n}(x)=\mathbf{1}_{\{x<0\}}(x)$. This contradiction proves Proposition 1, assuming that claim (24) holds.

Claim (24) follows from the proposition below because of (5).

Proposition 3. Suppose that two nonincreasing càdlàg functions $u, v: \mathbb{R} \rightarrow[0,1]$ satisfy

$$
\sum_{k=1}^{k_{0}} p_{k} g_{k} * Q_{1, k}(u)(x) \leq v(x) \leq \sum_{k=1}^{k_{0}} p_{k} g_{k} * Q_{2, k}(u)(x),
$$

where the $p_{k}$ are nonnegative integers such that $\sum_{k=1}^{k_{0}} p_{k}=1$ and the $g_{k}$ are distribution functions that satisfy the same assumptions as $p_{n, k}$ and $g_{n, k}$ in Section 2, and $Q_{1, k}$ and $Q_{2, k}$ are defined as in (4). Then

$$
L(v)>C \quad \Longrightarrow \quad L(u)>C .
$$

In order to prove Proposition 3, a few observations, notation, and lemmas are needed. Starting from $L(v)>C$, we obtain, by the definition of the Lyapunov function given in (6), that there exists an $x_{1} \in \mathbb{R}$ such that

$$
v\left(x_{1}\right) \leq \frac{1}{2} \quad \text { and } \quad l\left(v ; x_{1}\right)>\max \left\{C, L(v)-\frac{1}{4} \log m_{0}\right\} .
$$

By the definition of $l(v ; x)$ given in (7), we see that $v$ is small and flat at $x_{1}$ in the sense that

$$
1+\varepsilon:=\frac{v\left(x_{2}\right)}{v\left(x_{1}\right)}<1+\varepsilon_{1}
$$

and

$$
f_{0}:=v\left(x_{1}\right)<\left(\varepsilon_{1}-\varepsilon\right)^{1 / \log b} \mathrm{e}^{-C}<\frac{1}{2},
$$

where $x_{2}:=x_{1}-M$. Using bounds (25) and (28), we obtain

$$
\sum_{k=1}^{k_{0}} p_{k} g_{k} * Q_{1, k}(u)\left(x_{2}\right) \leq(1+\varepsilon) \sum_{k=1}^{k_{0}} p_{k} g_{k} * Q_{2, k}(u)\left(x_{1}\right),
$$

from which we will search for a flat piece in $u(x)$ where $u(x)$ is also small.

To control the value of $u(x)$, we derive here some preliminary estimates of $u(x)$ at $x_{1}$ and $x_{2}$, which will be used later to control the value of $u(x)$ at other places. For $i=1,2$, using the fact that $Q_{1, k}(u)\left(x_{i}-y\right)$ is positive and increasing in $y$ and then applying (25) and the fact that $\bar{g}_{k}(0) \geq 1-\varepsilon_{0}$ from assumption (MT1), we obtain

$$
\sum_{k=1}^{k_{0}} p_{k} Q_{1, k}(u)\left(x_{i}\right) \leq \sum_{k=1}^{k_{0}} p_{k} \frac{1}{\bar{g}_{k}(0)} \int_{0}^{\infty} Q_{1, k}(u)\left(x_{i}-y\right) \mathrm{d} g_{k}(y) \leq \frac{1}{1-\varepsilon_{0}} v\left(x_{i}\right) .
$$

This, together with the lower bound on $Q_{1, k}$ given in (16), the definition of $f_{0}$ given in (29), and the definition of $\varepsilon$ given in (28), implies that

$$
u\left(x_{1}\right) \leq \frac{f_{0}}{1-\varepsilon_{0}}
$$


and

$$
u\left(x_{2}\right) \leq \frac{1+\varepsilon}{1-\varepsilon_{0}} f_{0} .
$$

A finer estimate of $u\left(x_{2}\right)$ can be obtained and will be needed. First, using (31) and the lower bound on $Q_{1, k}$ given in (17), we obtain

$$
\left(\sum_{k=1}^{k_{0}} k p_{k}-c_{1} u\left(x_{2}\right)\right) u\left(x_{2}\right) \leq \frac{1+\varepsilon}{1-\varepsilon_{0}} f_{0} .
$$

By combining the first estimate of $u\left(x_{2}\right)$ given in (32), bound (29) on $f_{0}$, and the restriction (20), the coefficient multiplying $u\left(x_{2}\right)$ on the left-hand side of the above inequality is at least

$$
\begin{aligned}
\sum_{k=1}^{k_{0}} k p_{k}-c_{1} u\left(x_{2}\right) & \geq \sum_{k=1}^{k_{0}} k p_{k}-c_{1} \frac{1+\varepsilon}{1-\varepsilon_{0}} f_{0} \\
& \geq \sum_{k=1}^{k_{0}} k p_{k}-c_{1} \frac{1+\varepsilon_{1}}{1-\varepsilon_{0}}\left(\varepsilon_{1}-\varepsilon\right)^{1 / \log b} \mathrm{e}^{-C} \\
& \geq \sum_{k=1}^{k_{0}} k p_{k}-c_{1} \frac{1+\varepsilon_{1}}{1-\varepsilon_{0}} \varepsilon_{1}^{1 / \log b} \\
& \geq m_{0} .
\end{aligned}
$$

Therefore, we conclude that

$$
u\left(x_{2}\right) \leq \frac{1+\varepsilon}{m_{0}\left(1-\varepsilon_{0}\right)} f_{0}=\frac{1+\varepsilon}{m_{0}\left(1-\varepsilon_{0}\right)} v\left(x_{1}\right) .
$$

To control the flatness of $u(x)$, we define some more auxiliary variables and then state some lemmas. The constants $\delta=\kappa\left(\varepsilon_{1}-\varepsilon\right), \varepsilon^{\prime}=\varepsilon+\delta, \varepsilon^{\prime \prime}=\varepsilon+2 \delta$, and $\varepsilon^{(3)}=\varepsilon+3 \delta$ are defined to monitor the flatness change. Note that $\varepsilon, \varepsilon^{\prime}, \varepsilon^{\prime \prime}, \varepsilon^{(3)}<\varepsilon_{1}$ because $\kappa<\frac{1}{100}$. We somewhat simplify the argument in [3]. Set

$$
\begin{gathered}
y_{0}=\frac{1}{a} \log \frac{2 k_{0}}{\delta f_{0}}, \\
q=\inf \left\{y \geq \frac{1}{2} M: u\left(x_{2}-y\right)>\left(4 k_{0}\right)^{2} u\left(x_{1}-y\right)\right\},
\end{gathered}
$$

and

$$
r=y_{0} \wedge \begin{cases}q & \text { if } u\left(x_{2}-q\right)^{-} \geq\left(4 k_{0}\right) u\left(x_{1}-(q+M / 2)\right), \\ q-\frac{1}{2} M & \text { otherwise, }\end{cases}
$$

where $f(x)^{-}:=\lim _{y \rightarrow x-} f(y)$ is the left limit of $f$ at $x$. Intuitively, $q$ is used to denote the first nonflat place to the left of $x_{1}$. When $r<y_{0}, r$ is used to denote a nonflat interval, namely, it is easy to check that

$$
u\left(x_{2}-y\right) \geq\left(4 k_{0}\right) u\left(x_{1}-y\right) \quad \text { for all } y \in(r, r+M / 2] .
$$

We can now state the following sequence of lemmas, whose proofs will be discussed in the next subsection. The convention

$$
\int_{a}^{b} f(x) \mathrm{d} g(x)=\int_{(a, b]} f(x) \mathrm{d} g(x)
$$

for $a, b \in \mathbb{R}$ will be used throughout the rest of the paper. 
Lemma 5. Assume that (29) and (30) hold. Then

$$
\sum_{k=1}^{k_{0}} p_{k} \int_{-\infty}^{r} Q_{1, k}(u)\left(x_{2}-y\right) \mathrm{d} g_{k}(y) \leq\left(1+\varepsilon^{\prime}\right) \sum_{k=1}^{k_{0}} p_{k} \int_{-\infty}^{r} Q_{2, k}(u)\left(x_{1}-y\right) \mathrm{d} g_{k}(y) .
$$

Lemma 6. If (29) and (36) are satisfied, then there exist some $1 \leq k \leq k_{0}$ and $r^{\prime}$ such that

$$
\int_{-\infty}^{r^{\prime}} u\left(x_{2}-y\right) \mathrm{d} g_{k}(y) \leq\left(1+\varepsilon^{\prime \prime}\right) \int_{-\infty}^{r^{\prime}} u\left(x_{1}-y\right) \mathrm{d} g_{k}(y) .
$$

Moreover, $r^{\prime}$ is chosen such that $r^{\prime}>M$ implies that $r^{\prime}=r$.

Lemma 7. Suppose that (37) holds. Then

(a) $u\left(x_{2}-y_{1}\right) \leq\left(1+\varepsilon^{(3)}\right) u\left(x_{1}-y_{1}\right)$ for some $y_{1} \leq r^{\prime} \wedge M$, or

(b) $u\left(x_{2}-y_{1}\right) \leq\left(1+\varepsilon^{\prime \prime}-\delta \mathrm{e}^{a y_{1} / 8}\right) u\left(x_{1}-y_{1}\right)$ for some $y_{1} \in(M, r]$.

Lemma 6 and Lemma 7 are analogs of Lemma 3.5 and Proposition 3.2 of [3], respectively. Equipped with Lemma 7, we are ready to prove Proposition 3.

Proof of Proposition 3, assuming that Lemma 7 holds. We will compare $L(u)$ and $L(v)$ based on (33) and Lemma 7. As Lemma 7 suggests, it is necessary to consider two different cases.

Case (a). Assume that $u\left(x_{2}-y_{1}\right) \leq\left(1+\varepsilon^{(3)}\right) u\left(x_{1}-y_{1}\right)$ for some $y_{1} \leq r^{\prime} \wedge M$. Then, (33) implies that

$$
u\left(x_{1}-y_{1}\right) \leq u\left(x_{2}\right) \leq \frac{1+\varepsilon}{m_{0}\left(1-\varepsilon_{0}\right)} v\left(x_{1}\right) .
$$

Therefore, it follows from the definition of $l(u ; x)$ given in (7) that

$$
\begin{aligned}
l\left(u, x_{1}-y_{1}\right)-l\left(v, x_{1}\right) & \geq \log \frac{v\left(x_{1}\right)}{u\left(x_{1}-y_{1}\right)}+\log _{b} \frac{\varepsilon_{1}-\varepsilon^{(3)}}{\varepsilon_{1}-\varepsilon} \\
& \geq \log \frac{m_{0}\left(1-\varepsilon_{0}\right)}{1+\varepsilon}+\log _{b}(1-3 \kappa) \\
& \geq \log m_{0}-2\left(\varepsilon_{1}+\varepsilon_{0}\right)-\frac{6 \kappa}{\log b} \\
& \geq \frac{\log m_{0}}{2}
\end{aligned}
$$

where (21) guarantees the last inequality.

Case $(b)$. Assume that $u\left(x_{2}-y_{1}\right) \leq\left(1+\varepsilon^{\prime \prime}-\delta \mathrm{e}^{a y_{1} / 8}\right) u\left(x_{1}-y_{1}\right)$ for some $y_{1} \in(M, r]$. Then, by the definition of $r$ given in (34), if $y_{1} \in[j M,(j+1) M)$ then

$$
\begin{aligned}
u\left(x_{2}-\left(y_{1}-i M\right)\right) & \leq\left(4 k_{0}\right)^{2} u\left(x_{1}-\left(y_{1}-i M\right)\right) \\
& =\left(4 k_{0}\right)^{2} u\left(x_{2}-\left(y_{1}-(i+1) M\right)\right) \text { for } i=1, \ldots, j-1 .
\end{aligned}
$$

Thus,

$$
\begin{aligned}
u\left(x_{1}-y_{1}\right) & =u\left(x_{2}-\left(y_{1}-M\right)\right) \\
& \leq\left(4 k_{0}\right)^{2\left(\left\lfloor y_{1} / M\right\rfloor-1\right)} u\left(x_{2}-\left(y_{1}-\left\lfloor\frac{y_{1}}{M}\right\rfloor M\right)\right) \\
& \leq\left(4 k_{0}\right)^{2\left(y_{1} / M-1\right)} u\left(x_{2}-\left(y_{1}-\left\lfloor\frac{y_{1}}{M}\right\rfloor M\right)\right) .
\end{aligned}
$$


When $0 \leq y_{1}-\left\lfloor y_{1} / M\right\rfloor M \leq M / 2$,

$$
u\left(x_{2}-\left(y_{1}-\left\lfloor\frac{y_{1}}{M}\right\rfloor M\right)\right) \leq u\left(x_{2}-\frac{M}{2}\right) \leq\left(4 k_{0}\right)^{2} u\left(x_{1}-\frac{M}{2}\right)
$$

when $M / 2 \leq y_{1}-\left\lfloor y_{1} / M\right\rfloor M<M$,

$$
\begin{aligned}
u\left(x_{2}-\left(y_{1}-\left\lfloor\frac{y_{1}}{M}\right\rfloor M\right)\right) & \leq\left(4 k_{0}\right)^{2} u\left(x_{1}-\left(y_{1}-\left\lfloor\frac{y_{1}}{M}\right\rfloor M\right)\right) \\
& \leq\left(4 k_{0}\right)^{2} u\left(x_{2}-\frac{M}{2}\right) \\
& \leq\left(4 k_{0}\right)^{4} u\left(x_{1}-\frac{M}{2}\right) .
\end{aligned}
$$

The above inequalities and (33) imply that

$$
u\left(x_{1}-y_{1}\right) \leq\left(4 k_{0}\right)^{2 y_{1} / M+2} u\left(x_{1}-\frac{M}{2}\right) \leq\left(4 k_{0}\right)^{2 y_{1} / M+2} \frac{1+\varepsilon}{m_{0}\left(1-\varepsilon_{0}\right)} v\left(x_{1}\right) .
$$

Therefore, it follows that

$$
\begin{aligned}
l\left(u, x_{1}-y_{1}\right)-l\left(v, x_{1}\right) \geq & \log \frac{v\left(x_{1}\right)}{u\left(x_{1}-y_{1}\right)}+\log _{b} \frac{\varepsilon_{1}-\varepsilon^{\prime \prime}+\delta \mathrm{e}^{a y_{1} / 8}}{\varepsilon_{1}-\varepsilon} \\
\geq & \log \frac{m_{0}\left(1-\varepsilon_{0}\right)}{(1+\varepsilon)\left(4 k_{0}\right)^{2 y_{1} / M+2}}+\log _{b}\left(1-2 \kappa+\kappa \mathrm{e}^{a y_{1} / 8}\right) \\
\geq & \log m_{0}-2\left(\varepsilon_{0}+\varepsilon_{1}\right)-\frac{2 \log \left(4 k_{0}\right)}{M} y_{1}-2 \log \left(4 k_{0}\right) \\
& +\frac{\log \kappa+a y_{1} / 8}{\log b} .
\end{aligned}
$$

Rewrite the last term $a y_{1} / 8 \log b$ as $a y_{1} / 16 \log b+a y_{1} / 16 \log b$, use $y_{1} \geq M$ in one summand, and deduce that the above quantity is at least

$\log m_{0}-2\left(\varepsilon_{0}+\varepsilon_{1}+\log \left(4 k_{0}\right)\right)+\frac{\log \kappa}{\log b}+\frac{a M}{16 \log b}+y_{1}\left(\frac{a}{16 \log b}-\frac{2 \log \left(4 k_{0}\right)}{M}\right) \geq \frac{1}{2} \log m_{0}$, where (22) and (23) guarantee the last inequality.

To complete the argument, both cases imply that, by (7), (27), and the fact that $C=\log 2$,

$$
\log \frac{1}{u\left(x_{1}-y_{1}\right)} \geq l\left(u, x_{1}-y_{1}\right) \geq C+\frac{1}{2} \log m_{0} \geq \log 2,
$$

which implies that $u\left(x_{1}-y_{1}\right) \leq \frac{1}{2}$. Therefore, by the definition of $L(u)$ given in (6) and (27) again,

$$
L(u) \geq l\left(u, x_{1}-y_{1}\right) \geq l\left(v, x_{1}\right)+\frac{1}{2} \log m_{0} \geq L(v)+\frac{1}{4} \log m_{0} \geq L(v),
$$

from which (26) follows. Thus, the proof of Proposition 3 is complete. 


\subsection{Proofs of the lemmas}

Proof of Lemma 5. This lemma is to justify the flatness of the truncated integral. That is, we want to prove that mass from far away does not affect the value of the integral in a significant way. This is almost guaranteed by the exponential decay of $g_{n, k}(\cdot)$. However, we need to control the difference between $Q_{1, k}(u)\left(x_{2}-y\right)$ and $Q_{2, k}(u)\left(x_{1}-y\right)$, using the lower bound (16) on $Q_{1, k}(u)$ and the definition of $Q_{2, k}(u)$ given in (4). Two different cases will be presented separately.

Case (i): when $r<y_{0}$, (35) holds. Because of (30) and $\varepsilon<\varepsilon^{\prime}$, (36) will follow from

$$
\int_{r}^{\infty} Q_{1, k}(u)\left(x_{2}-y\right) \mathrm{d} g_{k}(y)-\left(1+\varepsilon^{\prime}\right) \int_{r}^{\infty} Q_{2, k}(u)\left(x_{1}-y\right) \mathrm{d} g_{k}(y) \geq 0
$$

for any $k \in\left\{1, \ldots, k_{0}\right\}$. To prove (39), because of (16), it suffices to show that

$$
\int_{r}^{\infty} u\left(x_{2}-y\right) \mathrm{d} g_{k}(y)-2 k_{0} \int_{r}^{\infty} u\left(x_{1}-y\right) \mathrm{d} g_{k}(y) \geq 0 .
$$

We split the left-hand side into three parts. First, by (35),

$$
\begin{aligned}
\frac{1}{2} \int_{r}^{r+M / 2} u\left(x_{2}-y\right) \mathrm{d} g_{k}(y)-2 k_{0} \int_{r}^{r+M / 2} u\left(x_{1}-y\right) \mathrm{d} g_{k}(y) \\
\quad=\int_{r}^{r+M / 2}\left(\frac{1}{2} u\left(x_{2}-y\right)-2 k_{0} u\left(x_{1}-y\right)\right) \mathrm{d} g_{k}(y) \\
\quad \geq 0 .
\end{aligned}
$$

Second, because of assumption (MT2) (the rapid decay of $\left.\bar{g}_{k}(\cdot)\right)$ and (18), we have

$$
\begin{aligned}
\frac{1}{2} \int_{r}^{r+M / 2} u\left(x_{2}-y\right) \mathrm{d} g_{k}(y)-2 k_{0} \int_{r+M / 2}^{r+M} u\left(x_{1}-y\right) \mathrm{d} g_{k}(y) \\
\quad \geq \frac{1}{4} u\left(x_{2}-r\right) \bar{g}_{k}(r)-2 k_{0} u\left(x_{2}-r\right) \bar{g}_{k}\left(r+\frac{1}{2} M\right) \\
\quad \geq\left(\frac{1}{4}-2 k_{0} \mathrm{e}^{-a M / 2}\right) u\left(x_{2}-r\right) \bar{g}_{k}(r) \\
\quad \geq 0 .
\end{aligned}
$$

Third, again because of assumption (MT2) (the rapid decay of $\left.\bar{g}_{k}(\cdot)\right)$ and (18), we have

$$
\begin{aligned}
\int_{r+M / 2}^{\infty} u\left(x_{2}-y\right) \mathrm{d} g_{k}(y)-2 k_{0} \int_{r+M}^{\infty} u\left(x_{1}-y\right) \mathrm{d} g_{k}(y) \\
\quad \geq \int_{r+M / 2}^{\infty} u\left(x_{2}-\left(y-\frac{M}{2}\right)\right) \mathrm{d} g_{k}(y)-2 k_{0} \int_{r}^{\infty} u\left(x_{2}-y\right) \mathrm{d} g_{k}(y+M) \\
\quad=\int_{r}^{\infty} u\left(x_{2}-y\right) \mathrm{d} g_{k}\left(y+\frac{M}{2}\right)-2 k_{0} \int_{r}^{\infty} u\left(x_{2}-y\right) \mathrm{d} g_{k}(y+M) \\
\geq\left(1-2 k_{0} \mathrm{e}^{-a M / 2}\right) \int_{r}^{\infty} u\left(x_{2}-y\right) \mathrm{d} g_{k}\left(y+\frac{M}{2}\right) \\
\geq 0 .
\end{aligned}
$$

Summing (42), (43), and (44) yields (40). Thus, (39) is verified in this case, and (36) holds. 
Case (ii): when $r=y_{0}$, (35) may not hold. The difference between the two sides of (36) is

$$
\begin{gathered}
\sum_{k=1}^{k_{0}} p_{k} \int_{-\infty}^{r} Q_{1, k}(u)\left(x_{2}-y\right) \mathrm{d} g_{k}(y)-\left(1+\varepsilon^{\prime}\right) \sum_{k=0}^{k_{0}} p_{k} \int_{-\infty}^{r} Q_{2, k}(u)\left(x_{1}-y\right) \mathrm{d} g_{k}(y) \\
\leq \sum_{k=1}^{k_{0}} p_{k} g_{k} * Q_{1, k}(u)\left(x_{2}\right)-\left(1+\varepsilon^{\prime}\right) \sum_{k=1}^{k_{0}} p_{k} \int_{-\infty}^{r} Q_{2, k}(u)\left(x_{1}-y\right) \mathrm{d} g_{k}(y) .
\end{gathered}
$$

Recall that $\varepsilon^{\prime}=\varepsilon+\delta$. Equation (30) implies that the above quantity is less than or equal to

$$
\begin{array}{r}
(1+\varepsilon) \sum_{k=1}^{k_{0}} p_{k} g_{k} * Q_{2, k}(u)\left(x_{1}\right)-\left(1+\varepsilon^{\prime}\right) \sum_{k=1}^{k_{0}} p_{k} \int_{-\infty}^{r} Q_{2, k}(u)\left(x_{1}-y\right) \mathrm{d} g_{k}(y) \\
=\left(1+\varepsilon^{\prime}\right) \sum_{k=1}^{k_{0}} p_{k} \int_{r}^{\infty} Q_{2, k}(u)\left(x_{1}-y\right) \mathrm{d} g_{k}(y)-\delta \sum_{k=1}^{k_{0}} p_{k} g_{k} * Q_{2, k}(u)\left(x_{1}\right) .
\end{array}
$$

Since $r=y_{0}=(1 / a) \log \left(2 k_{0} / \delta f_{0}\right)$, assumption (MT2) implies that $\bar{g}_{k}(r) \leq \mathrm{e}^{-a y_{0}}=\delta f_{0} / 2 k_{0}$. From $Q_{2, k}(u) \leq k_{0},(25)$, and (29), it follows that the above quantity again does not exceed

$$
\left(1+\varepsilon^{\prime}\right) k_{0} \frac{\delta f_{0}}{2 k_{0}}-\delta f_{0} \leq 0
$$

So (36) is proved in this case. This completes the proof of the lemma.

Proof of Lemma 6. By the definition of $q$, when $q>M / 2$, we have

$$
u\left(x_{2}-y\right) \leq\left(4 k_{0}\right)^{2} u\left(x_{1}-y\right) \text { for } y \in[M / 2, q] .
$$

Similarly to (38), for any $y \leq r \leq q$, we obtain

$$
u\left(x_{2}-y\right) \leq u\left(x_{2}-r\right) \leq\left(4 k_{0}\right)^{2 r / M+2} u\left(x_{2}\right) .
$$

Since $r \leq y_{0}=(1 / a) \log \left(2 k_{0} / \delta f_{0}\right)$, using (32), the above is at most

$$
\left(4 k_{0}\right)^{2 y_{0} / M+2} \frac{1+\varepsilon}{1-\varepsilon_{0}} f_{0}<\frac{2\left(4 k_{0}\right)^{2}}{1-\varepsilon_{0}}\left(4 k_{0}\right)^{(2 / a M) \log \left(2 k_{0} / \delta f_{0}\right)} f_{0}=\frac{2\left(4 k_{0}\right)^{2}}{1-\varepsilon_{0}}\left(\frac{2 k_{0}}{\delta f_{0}}\right)^{2 \log \left(4 k_{0}\right) / a M} f_{0} \text {. }
$$

Note that $2 \log \left(4 k_{0}\right) / a M<\frac{1}{2}$ from (18). Applying the bound on $f_{0}$ given in (29), the above quantity is at most

$$
\frac{2\left(4 k_{0}\right)^{2}}{1-\varepsilon_{0}} \frac{\sqrt{2 k_{0}} f_{0}^{1 / 2}}{\delta^{1 / 2}}=\frac{8\left(2 k_{0}\right)^{5 / 2} f_{0}^{1 / 2}}{\left(1-\varepsilon_{0}\right) \delta^{3 / 2}} \delta<\frac{8\left(2 k_{0}\right)^{5 / 2}\left(\varepsilon_{1}-\varepsilon\right)^{1 / 2 \log b-3 / 2}}{\left(1-\varepsilon_{0}\right) \kappa^{3 / 2}} \delta .
$$

Therefore, it follows from (19) that

$$
u\left(x_{2}-y\right) \leq \frac{1}{2 c_{1}} \delta \text { for any } y \leq r .
$$

This, combined with (17), implies that, for any $1 \leq k \leq k_{0}$ and $y \leq r_{1}$,

$$
\begin{aligned}
Q_{1, k}(u)\left(x_{2}-y\right) & \geq k u\left(x_{2}-y\right)-c_{1}\left(u\left(x_{2}-y\right)\right)^{2} \\
& =k u\left(x_{2}-y\right)\left(1-\frac{c_{1}}{k} u\left(x_{2}-y\right)\right) \\
& \geq k u\left(x_{2}-y\right)\left(1-\frac{1}{2} \delta\right) .
\end{aligned}
$$


We have

$$
\begin{aligned}
& \frac{\sum_{k=1}^{k_{0}} k p_{k} \int_{-\infty}^{r} u\left(x_{2}-y\right) \mathrm{d} g_{k}(y)}{\sum_{k=1}^{k_{0}} k p_{k} \int_{-\infty}^{r} u\left(x_{1}-y\right) \mathrm{d} g_{k}(y)} \\
& \quad \leq\left(\frac{1}{1-\delta / 2}\right) \frac{\sum_{k=1}^{k_{0}} p_{k} \int_{-\infty}^{r} Q_{1, k}(u)\left(x_{2}-y\right) \mathrm{d} G_{k}(y)}{\sum_{k=1}^{k_{0}} p_{k} \int_{-\infty}^{r} Q_{2, k}(u)\left(x_{1}-y\right) \mathrm{d} G_{k}(y)} \\
& \quad \leq \frac{1+\varepsilon^{\prime}}{1-\delta / 2} \\
& \quad \leq 1+\varepsilon^{\prime \prime} .
\end{aligned}
$$

where the first inequality follows from (46) and the definition of $Q_{2, k}(u)$ given in (4), and the second inequality follows from (36). If the conclusion of the lemma does not hold, i.e. for all $1 \leq k \leq k_{0}$,

$$
\int_{-\infty}^{r} u\left(x_{2}-y\right) \mathrm{d} g_{k}(y)>\left(1+\varepsilon^{\prime \prime}\right) \int_{-\infty}^{r} u\left(x_{1}-y\right) \mathrm{d} g_{k}(y)
$$

we obtain a contradiction to (47). This completes the proof of Lemma 6 in the case $q>M / 2$.

When $q=M / 2$ and $u\left(x_{2}-M / 2\right) \leq 4 k_{0} u\left(x_{2}\right)$, with (32), we still have, for $y \leq r \leq q$,

$$
u\left(x_{2}-y\right) \leq u\left(x_{2}-r\right) \leq 4 k_{0} u\left(x_{2}\right) \leq \frac{8 k_{0} f_{0}}{\left(1-\varepsilon_{0}\right) \delta} \delta .
$$

Using the bound on $f_{0}$ given in (29) and (19), the above is at most

$$
\frac{8 k_{0}\left(\varepsilon_{1}-\varepsilon\right)^{1 / \log b-1}}{\left(1-\varepsilon_{0}\right) \kappa} \delta \leq \frac{1}{2 c_{1}} \delta .
$$

Thus, (45) holds. Repeating the argument below (45), we obtain Lemma 6 in this case.

When $q=M / 2$, but $u\left(x_{2}-M / 2\right)>\left(4 k_{0}\right) u\left(x_{2}\right)$, we truncate (36) before transforming this case to the previous case. Define

$$
r^{\prime}=\inf \left\{y \geq 0: u\left(x_{2}-y\right)>4 k_{0} u\left(x_{2}\right)\right\} .
$$

Then $0 \leq r^{\prime}<M / 2$ and $u\left(x_{2}-r^{\prime}\right) \leq 4 k_{0} u\left(x_{2}\right)$. By the monotonicity of $u, u\left(x_{2}-y\right) \geq$ $4 k_{0} u\left(x_{1}-y\right)$ for $y \in\left(r^{\prime}, r\right]$. Therefore, for $1 \leq k \leq k_{0}$,

$$
\begin{aligned}
\int_{r^{\prime}}^{r} & Q_{1, k}(u)\left(x_{2}-y\right) \mathrm{d} g_{k}(y)-\left(1+\varepsilon^{\prime}\right) \int_{r^{\prime}}^{r} Q_{2, k}(u)\left(x_{1}-y\right) \mathrm{d} g_{k}(y) \\
& \geq \int_{r^{\prime}}^{r} u\left(x_{2}-y\right) \mathrm{d} g_{k}(y)-2 \int_{r^{\prime}}^{r} k_{0} u\left(x_{1}-y\right) \mathrm{d} g_{k}(y) \\
& =\int_{r^{\prime}}^{r}\left(u\left(x_{2}-y\right)-2 k_{0} u\left(x_{1}-y\right)\right) \mathrm{d} g_{k}(y) \\
& \geq 0
\end{aligned}
$$

which, together with (36), yields the truncated inequality

$$
\sum_{k=1}^{k_{0}} p_{k} \int_{-\infty}^{r^{\prime}} Q_{1, k}(u)\left(x_{2}-y\right) \mathrm{d} g_{k}(y) \leq\left(1+\varepsilon^{\prime}\right) \sum_{k=1}^{k_{0}} p_{k} \int_{-\infty}^{r^{\prime}} Q_{2, k}(u)\left(x_{1}-y\right) \mathrm{d} g_{k}(y) .
$$

This is an analog of (36) with $r$ replaced by $r^{\prime}$, and $u\left(x_{2}-r^{\prime}\right) \leq 4 k_{0} u\left(x_{2}\right)$. Replacing $r$ by $r^{\prime}$ in the argument starting from (48), we complete the proof of Lemma 6 in all cases. 
With assumption (MT2), the proof of [3, Proposition 3.2] carries over (with some change of notation) to the proof of Lemma 7, assuming that Lemma 6 holds.

Proof of Lemma 7. This will be proved by contradiction. Assume that neither (a) nor (b) of Lemma 7 holds, i.e.

(a) $u\left(x_{2}-y\right)>\left(1+\varepsilon^{(3)}\right) u\left(x_{1}-y\right)$ for all $y \leq r^{\prime} \wedge M$, and

(b) $u\left(x_{2}-y\right)>\left(1+\varepsilon^{\prime \prime}-\delta \mathrm{e}^{a y / 8}\right) u\left(x_{1}-y\right)$ for all $y \in\left(M, r^{\prime}\right]$.

If $r^{\prime} \leq M$ then only ( $\left.\overline{\mathrm{a}}\right)$ holds and it implies that

$$
\int_{-\infty}^{r^{\prime}} u\left(x_{2}-y\right) \mathrm{d} g_{k}(y)>\left(1+\varepsilon^{(3)}\right) \int_{-\infty}^{r^{\prime}} u\left(x_{1}-y\right) \mathrm{d} g_{k}(y) .
$$

Since $\varepsilon^{(3)}=\varepsilon^{\prime \prime}+\delta>\varepsilon^{\prime \prime}$, this contradicts (37). So we are done. If $r^{\prime}>M$ then $r^{\prime}=r$, and $(\overline{\mathrm{a}})$ and $(\overline{\mathrm{b}})$ imply that

$$
\int_{-\infty}^{M} u\left(x_{2}-y\right) \mathrm{d} g_{k}(y)>\left(1+\varepsilon^{(3)}\right) \int_{-\infty}^{M} u\left(x_{1}-y\right) \mathrm{d} g_{k}(y)
$$

and

$$
\int_{M}^{r} u\left(x_{2}-y\right) \mathrm{d} g_{k}(y)>\int_{M}^{r}\left(1+\varepsilon^{\prime \prime}-\delta \mathrm{e}^{a y / 8}\right) u\left(x_{1}-y\right) \mathrm{d} g_{k}(y) .
$$

Summing the above two inequalities yields

$$
\begin{aligned}
\int_{-\infty}^{r} u\left(x_{2}-y\right) \mathrm{d} g_{k}(y)> & \left(1+\varepsilon^{\prime \prime}\right) \int_{-\infty}^{r} u\left(x_{1}-y\right) \mathrm{d} g_{k}(y) \\
& +\delta\left[\int_{-\infty}^{M} u\left(x_{1}-y\right) \mathrm{d} g_{k}(y)-\int_{M}^{r} \mathrm{e}^{a y / 8} u\left(x_{1}-y\right) \mathrm{d} g_{k}(y)\right] .
\end{aligned}
$$

We claim that

$$
\int_{-\infty}^{M} u\left(x_{1}-y\right) \mathrm{d} g_{k}(y)-\int_{M}^{r} \mathrm{e}^{a y / 8} u\left(x_{1}-y\right) \mathrm{d} g_{k}(y) \geq 0,
$$

which will imply a contradiction of (36) and complete the proof. It thus remains to prove claim (49). Since $q \geq r>M / 2$, we have $u\left(x_{2}-y\right) \leq\left(4 k_{0}\right)^{2} u\left(x_{1}-y\right)$ for all $y \in[M / 2, r]$. By (18), we can bound the second integral on the left-hand side of the above inequality as follows:

$$
\begin{aligned}
\int_{M}^{r} \mathrm{e}^{a y / 8} u\left(x_{1}-y\right) \mathrm{d} g_{k}(y) & =\sum_{l=1}^{\infty} \int_{l M}^{l M+M} \mathrm{e}^{a y / 8} u\left(x_{1}-y\right) \mathbf{1}_{\{y \leq r\}} \mathrm{d} g_{k}(y) \\
& \leq \sum_{l=1}^{\infty} \int_{l M}^{l M+M} \mathrm{e}^{a l M / 8+a M / 8}\left(4 k_{0}\right)^{2 l+2} u\left(x_{1}-\frac{M}{2}\right) \mathrm{d} g_{k}(y) \\
& \leq \sum_{l=1}^{\infty} \mathrm{e}^{a l M / 8+a M / 8}\left(4 k_{0}\right)^{2 l+2} u\left(x_{1}-\frac{M}{2}\right) \bar{g}_{k}(l M) \\
& \leq \sum_{l=1}^{\infty} \mathrm{e}^{a l M / 8+a M / 8}\left(4 k_{0}\right)^{2 l+2} u\left(x_{1}-\frac{M}{2}\right) \mathrm{e}^{-a l M+a M / 2} \bar{g}_{k}\left(\frac{M}{2}\right) \\
& \leq \frac{1}{4} u\left(x_{1}-\frac{M}{2}\right) \bar{g}_{k}\left(\frac{M}{2}\right) .
\end{aligned}
$$


As the last term does not exceed

$$
\int_{M / 2}^{M} u\left(x_{1}-y\right) \mathrm{d} g_{k}(y) \leq \int_{-\infty}^{M} u\left(x_{1}-y\right) \mathrm{d} g_{k}(y),
$$

the proof of (49), and, thus, of Lemma (7), is complete.

\section{Tightness for identical marginals}

In this section we discuss the tightness problem in the case when all the marginal distributions at the same level are the same, i.e. $g_{n, k}(\cdot)=g_{n}(\cdot)$ does not depend on the number of offspring. Compared with the assumptions made in Section 2, we relax the bounded support assumption (B1) on $p_{n, k}$, at the price of a uniform marginal assumption on $G_{n, k}$ (see (MT0') below). Namely, we make the following assumptions.

$\left(\mathrm{B}^{\prime}\right)$ There exist positive real numbers $m_{0}$ and $m_{1}$ such that $\inf _{n}\left\{\sum_{k=1}^{\infty} k p_{n, k}\right\}>m_{0}>1$ and $\sup _{n} \sum_{k=1}^{\infty} k^{2} p_{n, k}<m_{1}$.

$\left(\mathrm{MT0}^{\prime}\right) g_{n, k}(\cdot)=g_{n}(\cdot)$ for all $k \geq 1$.

$\left(\mathrm{MT}^{\prime}\right)$ For some fixed $\varepsilon_{0}<\frac{1}{4} \log m_{0} \wedge 1$, there exists an $x_{0}$ such that $\bar{g}_{n}\left(x_{0}\right) \geq 1-\varepsilon_{0}$ for all $n$, where $\bar{g}_{n}(x)=1-g_{n}(x)$. By shifting, we will assume that $x_{0}=0$, that is, $\bar{g}_{n}(0) \geq 1-\varepsilon_{0}$.

$\left(\mathrm{MT}^{\prime}\right)$ There exist $a>0$ and $M_{0}>0$ such that $\bar{g}_{n}(x+M) \leq \mathrm{e}^{-a M} \bar{g}_{n}(x)$ for all $n$ and $M>M_{0}, x \geq 0$.

$\left(\mathrm{GT}^{\prime}\right)$ For any $\eta_{1}>0$, there exists a $B>0$ such that $G_{n, k}(B, \ldots, B) \geq 1-\eta_{1}$ and $\bar{g}_{n}(-B) \geq$ $1-\eta_{1}$ for all $n$ and $k$.

Then we still have the following tightness result.

Theorem 2. Under assumptions $\left(B 1^{\prime}\right),\left(M T 0^{\prime}\right),\left(M T 1^{\prime}\right),\left(M T 2^{\prime}\right)$, and $\left(G T^{\prime}\right)$, the family of recentered maxima distributions $\left\{F_{n}\left(\cdot-\operatorname{Med}\left(F_{n}\right)\right)\right\}$ is tight.

Since the proof is similar to that of Theorem 1, we only sketch the details. The argument is based on the recursion inequality

$$
g_{m} *\left(\sum_{k=1}^{\infty} p_{m, k} Q_{1, k}\left(\bar{F}_{n}^{m+1}\right)\right)(x) \leq \bar{F}_{n}^{m}(x) \leq g_{m} *\left(\sum_{k=1}^{\infty} p_{m, k} Q_{2, k}\left(\bar{F}_{n}^{m+1}\right)\right)(x)
$$

another form of (5) under assumption (MT0'), where $Q_{1, k}$ and $Q_{2, k}$ are defined as (4). Set

$$
Q_{m,(1)}(u)=\sum_{k=1}^{\infty} p_{m, k} Q_{1, k}(u)
$$

and

$$
Q_{m,(2)}(u)=\sum_{k=1}^{\infty} p_{m, k} Q_{2, k}(u) .
$$

Although the difference between $Q_{1, k}$ and $Q_{2, k}$ gets larger as $k$ increases, the weighted functions $Q_{m,(1)}$ and $Q_{m,(2)}$ still behave nicely and we have the following analog of Lemma 4. 
Lemma 8. Let $Q_{m,(1)}$ and $Q_{m,(2)}$ be defined as in (50) and (51), respectively. Then it follows from assumption $\left(B^{\prime}\right)$ that

$$
Q_{m,(1)}(u)>u
$$

and

$$
Q_{m,(2)}(u)-c_{2} u^{2} \leq Q_{m,(1)}(u) \leq Q_{m,(2)}(u) \leq \sqrt{m_{1}} u .
$$

Lemma 5 relies on the facts that $Q_{1, k}(u) \geq u$ and $Q_{2, k}(u) \leq k_{0} u$, and Lemma 6 relies on the fact that $Q_{1, k}(u) \geq Q_{2, k}(u)-c_{1} u^{2}$. Therefore, with modifications of $q$ and $r$, we can prove analogs of Lemmas 5 and 6 due to the bounds in Lemma 8. An analog of Proposition 3 then follows. Proposition 1 and Corollary 1 hold under the new assumptions in this section.

Assumption $\left(\mathrm{GT}^{\prime}\right)$ plays a similar role as $(\mathrm{GT})$ in connecting the left and right tail behaviors. Specifically, it guarantees Lemma 2, Lemma 3, and Proposition 2 under the new settings. Theorem 2 follows immediately as Theorem 1 .

\section{Remarks}

We believe that our assumptions (the bounded support assumption (B1) on $p_{n, k}$ in Section 2 and the uniform marginal assumption $\left(\mathrm{MT}^{\prime}\right)$ on $G_{n, k}$ in Section 5) are only technical, and we are not aware of any natural examples for which tightness does not hold without these assumptions. Heuristically, the faster the number of particles grows, the tighter the maximum is around its median. Therefore, we believe that these technical assumptions can possibly be removed, although the exact argument in the paper does not work directly without these assumptions.

\section{Acknowledgements}

I would like to thank my advisor, Ofer Zeitouni, for posing this problem and guiding me through this work. I also thank the anonymous referee for valuable comments. This research was partially supported by NSF grant DMS-0804133.

\section{References}

[1] Addario-Berry, L. And Reed, B. A. (2009). Minima in branching random walks. Ann. Prob. 37, $1044-1079$.

[2] Bramson, M. D. (1978). Maximal displacement of branching Brownian motion. Commun. Pure Appl. Math. 31, 531-581.

[3] Bramson, M. and Zeitouni, O. (2009). Tightness for a family of recursion equations. Ann. Prob. 37, 615-653.

[4] Bramson, M. and Zeitouni, O. (2012). Tightness of the recentered maximum of the two-dimensional discrete Gaussian free field. Commun. Pure Appl. Math. 65, 1-20.

[5] Dekking, F. M. And Host, B. (1991). Limit distributions for minimal displacement of branching random walks. Prob. Theory Relat. Fields 90, 403-426. 